\title{
Effect of congenital heart disease on neurodevelopmental outcomes within multiple-gestation births
}

\author{
Amy H. Schultz, MD, a Gail P. Jarvik, MD, PhD, ${ }^{\text {h }}$ Gil Wernovsky, MD, Judy Bernbaum, MD, ${ }^{\text {b }}$ Robert R. Clancy, MD, ${ }^{c}$ \\ Jo Ann D'Agostino, CRNP, ${ }^{\text {b }}$ Marsha Gerdes, PhD, ${ }^{d}$ Donna McDonald-McGinn, MS, CGC, ${ }^{\text {e }}$ Susan C. Nicolson, MD, ${ }^{g}$ \\ Thomas L. Spray, MD, Elaine Zackai, MD, ${ }^{f}$ and J. William Gaynor, $\mathrm{MD}^{f}$
}

From the Divisions of Cardiology, ${ }^{\mathrm{a}}$ General Pediatrics, ${ }^{\mathrm{b}}$ Neurology, ${ }^{\mathrm{c}}$ Psychology, ${ }^{\mathrm{d}}$ Genetics, ${ }^{\mathrm{e}}$ and Cardiothoracic Surgery, ${ }^{\mathrm{f}}$ and the Department of Anesthesia and Critical Care Medicine, ${ }^{\mathrm{g}}$ The Children's Hospital of Philadelphia and the University of Pennsylvania School of Medicine, Philadelphia, Pa, and the Division of Medical Genetics, ${ }^{\mathrm{h}}$ Department of Medicine, University of Washington, Seattle, Wash.

Supported by an American Heart Association National Grant-in-Aid (9950480N), the Pew Biomedical Scholar Program, the Fannie E. Rippel Foundation and NIH T32HL07915 (Dr Schultz).

Received for publication May 16, 2005; revisions received June 29, 2005; accepted for publication July 12, 2005.

Address for reprints: Amy H. Schultz, MD, Division of Cardiology, Children's Hospital and Regional Medical Center, 4800 Sand Point Way, W-4841, Seattle, WA 98105 (Email: ahschultz@uwalumni.com).

J Thorac Cardiovasc Surg 2005;130:1511-6 $0022-5223 / \$ 30.00$

Copyright (c) 2005 by The American Association for Thoracic Surgery

doi:10.1016/j.jtcvs.2005.07.040
Objectives: We sought to assess the effect of congenital heart disease requiring surgical intervention with cardiopulmonary bypass at 6 months of age or less on developmental outcomes and growth at 1 year of age while controlling for socioeconomic status, prematurity, home environment, and parental intelligence.

Methods: We performed within-family comparison of 11 multiple-gestation births in which one child had congenital heart disease. At 1 year of age, the Bayley Scales of Infant Development II were administered, and growth parameters were assessed. Paired comparisons were made by using fixed effects regression conditioned on family.

Results: The multiple-gestation subjects were mildly premature on average (mean gestational age, $35.4 \pm 3.0$ weeks). At 1 year of age, children with congenital heart disease scored lower on the Mental Development Index $(85.0 \pm 19.3$ vs $93.9 \pm$ $16.0, P=.037)$ and the Psychomotor Development Index (76.6 \pm 16.9 vs $91.3 \pm$ $14.9, P=.015)$ on the Bayley Scales of Infant Development II than did their siblings without congenital heart disease. There were no differences between siblings in weight, height, or head circumference.

Conclusions: The presence of congenital heart disease requiring surgical intervention with cardiopulmonary bypass at 6 months of age or less is associated with a deficit in developmental achievement at 1 year of age, as measured by using the Bayley Scales of Infant Development II.

$\mathrm{N}$ eurocognitive outcomes after surgical intervention for congenital heart disease (CHD) in infancy are the focus of great clinical interest and much research. As mortality among children with CHD has decreased, neurodevelopmental impairment has been identified as one of the most significant morbidities among survivors of CHD operations. Multiple studies have shown that in comparison with general population norms, survivors of surgical intervention for complex CHD typically have lower scores on neuropsychological testing, with performance scores often more impaired than verbal scores. ${ }^{1-4}$ However, comparison of children with CHD with published norms for the general population is complicated by the potential confounding effects of socioeconomic status (SES), parental intelligence, home environment, and prematurity, all of which highly influence developmental outcome. ${ }^{1,5-9}$ To isolate the effect of CHD and its management on neurodevelopmental outcomes at 1 year of age, we evaluated multiple-gestation births in which one child had CHD. This study design affords a unique opportunity to match study subjects and sibling control subjects for familial factors, including SES, parental intelligence, home environment, and gestational age.

\section{Methods}

\section{Patient Population}

Between September 1998 and April 2003, 550 infants with CHD undergoing surgical intervention with cardiopulmonary bypass (CPB) at 188 days (6 months) of age or less were 


\author{
Abbreviations and Acronyms \\ $\mathrm{CHD}=$ congenital heart disease \\ $\mathrm{CPB}=$ cardiopulmonary bypass \\ $\mathrm{DHCA}=$ deep hypothermic circulatory arrest \\ HLHS = hypoplastic left heart syndrome \\ MDI = Mental Development Index \\ PDI = Psychomotor Development Index \\ SES = socioeconomic status
}

enrolled in a single-institution study of the association between apolipoprotein E genotype and postoperative neurodevelopmental dysfunction. ${ }^{4}$ Exclusion criteria included (1) multiple congenital anomalies, (2) recognizable genetic or phenotypic syndrome other than chromosome 22q11 microdeletion, and (3) language other than English spoken in the home.

Among this cohort, subjects who were the product of a multiple gestation were identified; this group forms the study population for this report. Subjects with microdeletion of chromosome $22 \mathrm{q} 11$ were excluded from this study. Siblings of the same gestation were recruited to participate in the follow-up evaluation. Neonatal records of both the subjects with CHD and their siblings were reviewed for information about the pregnancy and perinatal course. Gestational age was recorded in completed weeks by best obstetric estimate.

The Institutional Review Board at The Children's Hospital of Philadelphia approved the study. Written informed consent was obtained from the parent or guardian. The funding agencies had no role in data collection, interpretation, or analysis or in the preparation of the manuscript.

\section{Intraoperative Management}

Operations were performed by 3 cardiac surgeons with a dedicated team of cardiac anesthesiologists. Alpha-stat blood gas management was used. Deep hypothermic circulatory arrest (DHCA) was used at the surgeon's discretion. Before DHCA, patients were cooled to a nasopharyngeal temperature of $18^{\circ} \mathrm{C}$ by a combination of core cooling on CPB and topical hypothermia. Modified ultrafiltration was performed in all patients.

\section{One-Year Follow-up Evaluation}

A study follow-up visit was conducted at 12 months of age, corrected for prematurity, plus or minus 2 weeks. Siblings were assessed on the same day. Personnel involved in the evaluation were not blinded to CHD status. One of 3 specialists in developmental pediatrics measured growth parameters and performed a neurologic examination. The same investigator evaluated all children in a family. Length was determined by using a supine board. Neurologic findings were classified as normal, suspect, or abnormal. A single psychologist administered the Bayley Scales of Infant Development II. Each child with CHD was evaluated by a genetic dysmorphologist to identify children with genetic syndromes that might not have been apparent earlier in infancy. Children were eliminated if the geneticist believed there were marked dysmorphisms, even if no named syndrome was diagnosed. SES, as measured by the Hollingshead Index, ${ }^{10}$ and ethnicity were determined by means of parental questionnaire.
The Bayley Scales of Infant Development II yields 2 scores: the Mental Development Index (MDI) and the Psychomotor Development Index (PDI). Each of these scores has a mean of 100 and a standard deviation of 15 in the general population. The MDI assesses memory, problem solving, early number concepts, generalization, expressive and receptive language, and social skills. The PDI evaluates control of gross motor functioning, including crawling and walking, as well as fine motor skills necessary for prehension, use of writing instruments, and imitation of hand movements.

\section{Data Analysis and Statistical Methods}

As a measure of complexity of CHD, patients were categorized according to a previously described classification that has been shown to be predictive of postoperative mortality. ${ }^{11}$ Class I includes patients whose repair achieves a biventricular circulation and in whom there is no preoperative arch obstruction, class II includes patients whose repair achieves a biventricular circulation with arch obstruction, class III includes patients whose repair achieves a single-ventricle circulation without arch obstruction, and class IV includes patients whose repair achieves a singleventricle circulation with arch obstruction.

Descriptive statistics were used to characterize the study population and other subsets of the larger cohort. Comparisons were made across groups by using $t$ tests, contingency tables, or Wilcoxon rank sum tests. Exact methods were used for contingency tables when any expected value was less than 5 . Within the multiple-gestation subjects, the distribution of MDI and PDI scores and growth parameters were inspected, and adequate symmetry was believed to be present to allow the use of parametric tests. Fixed effects regression conditioned on family group was used to make paired comparisons between subjects with CHD and their siblings. This strategy allowed incorporation of the data from both siblings in the sets of triplets. Statistical analyses were performed using STATA 7.0 (College Station, Tex).

The relationship between apolipoprotein E genotype and neurodevelopmental outcome was not explored in the multiplegestation cohort. The allele associated with increased risk of neurodevelopmental delay, $\epsilon 2$, had an allele frequency of approximately $8 \%$ in the larger cohort. Thus only 1 or 2 multiplegestation subjects with CHD and the $\epsilon 2$ allele could be expected in this study cohort, a number too small for any meaningful relationships to be identified.

\section{Results}

\section{Study Population}

Among 550 subjects enrolled in the larger cohort, 30 subjects (29 families) were the product of a multiple gestation. In 10 families one or more children died before 1 year of age (in 8 families the child with CHD died, in 1 the sibling without CHD died, and in 1 both members of a twin pair are known to have died). Three families were excluded because of microdeletion of chromosome $22 \mathrm{q} 11$ or diagnosis of a genetic syndrome in the child with CHD at the follow-up visit. One family was excluded because of the presence of CHD in both members of the twin pair. In 11 (73\%) of 15 remaining families ( 9 twin sets and 2 triplet sets), both the 
TABLE 1. Comparison of multiple-gestation subjects included versus excluded from the comparison of outcomes at 1 year of age

\begin{tabular}{|c|c|c|c|}
\hline Parameter & $\begin{array}{l}\text { Multiple births included in } \\
\text { study }(n=11)\end{array}$ & $\begin{array}{l}\text { Multiple births not included } \\
\text { in study }(\mathrm{n}=19)\end{array}$ & $P$ value \\
\hline \multicolumn{4}{|l|}{ Demographic } \\
\hline Female sex & $3(27.2 \%)$ & $10(52.6 \%)$ & .26 \\
\hline Race & & & .68 \\
\hline White, not Hispanic & $10(90.9 \%)$ & $13(68.4 \%)$ & \\
\hline Black, not Hispanic & $1(9.1 \%)$ & $3(15.8 \%)$ & \\
\hline Hispanic & & $2(10.5 \%)$ & \\
\hline Other & & $1(5.3 \%)$ & \\
\hline \multicolumn{4}{|l|}{ Perinatal information } \\
\hline Mean gestational age, wk (range) & $35.4 \pm 3.0(30-38)$ & $34.8 \pm 3.0(28-38)$ & .62 \\
\hline Term ( $\geq 37$ wk EGA) & $6(45.5 \%)$ & $7(36.8 \%)$ & .45 \\
\hline Type of delivery & & & .10 \\
\hline Vaginal & $1(9.1 \%)$ & $8(42.1 \%)$ & \\
\hline Cesarean section & $10(90.9 \%)$ & $11(57.9 \%)$ & \\
\hline Mean birth weight, kg (range) & $2.46 \pm 0.65(1.26-3.15)$ & $2.10 \pm 0.70(0.74-3.30)$ & .18 \\
\hline \multicolumn{4}{|l|}{$\mathrm{CHD}$ and surgery } \\
\hline CHD class & & & .016 \\
\hline Class I & $8(72.7 \%)$ & $3(15.8 \%)$ & \\
\hline Class II & $0(0 \%)$ & $4(21.1 \%)$ & \\
\hline Class III & $1(9.1 \%)$ & $3(15.8 \%)$ & \\
\hline Class IV & $2(18.2 \%)$ & $9(47.4 \%)$ & \\
\hline Median age at operation, $d$ (range) & $7(1-143)$ & $6(1-178)$ & .50 \\
\hline Operation at $\leq 30 \mathrm{~d}$ of age & $6(54.5 \%)$ & $13(68.4 \%)$ & .70 \\
\hline No. operations with CPB at $\leq 1$ y of age & & & 67 \\
\hline One & $9(81.8 \%)$ & $13(68.4 \%)$ & \\
\hline Two & $2(18.2 \%)$ & $6(31.6 \%)$ & \\
\hline
\end{tabular}

$E G A$, Estimated gestational age; $C H D$, congenital heart disease.

subject with CHD and his or her sibling or siblings were evaluated. Reasons for failure to return for 1-year follow-up included acute illness on the testing day, distance, inability to contact, and lack of awareness by the study staff that a child with CHD was a twin. In this last case, the child with CHD returned, but the sibling did not. These families included 3 subjects with hypoplastic left heart syndrome (HLHS) or HLHS variant and 1 subject with ventricular septal defect and coarctation.

Compared with the singleton births in the larger cohort, the multiple-gestation subjects included in this study were more premature $(35.4 \pm 3.0$ vs $38.5 \pm 2.1$ weeks, $P<$ .001 ), had a lower birth weight ( $2.46 \pm 0.65$ vs $3.14 \pm 0.62$ $\mathrm{kg}, P<.001)$, were more likely to be delivered by means of cesarean section $(10 / 11$ vs $136 / 506, P<.001)$, and had a higher SES (Hollingshead Index: $4.5 \pm 0.7$ vs $3.9 \pm 1.2$, $P=.009)$ than the study subjects who were the product of singleton pregnancies. Mortality in the first year of life was $9(30 \%)$ of 30 among all multiple gestations compared with $39(7.5 \%)$ of 520 for all singleton births $(P<.001)$.

Baseline characteristics of the included and not included multiple-gestation subjects are shown in Table 1. The multiple-gestation subjects not included had more complex CHD than those included, which is not surprising because 9 of those not included died before 1 year of age. Further descriptive information on the multiplegestation subjects with CHD is shown in Table 2. Four pregnancies were known to be the product of in vitro fertilization; 2 mothers conceived after pharmacologic ovarian stimulation.

\section{Comparison of Outcome Measures}

The primary outcomes of the study were the MDI and PDI scores on the Bayley Scales of Infant Development II at 1 year of age. Comparison of MDI and PDI by family are shown in Figures 1 and 2. Table 3 presents summary statistics for all outcome measures. Figures 1 and 2 show that children with CHD scored lower than their siblings on the MDI in $8(73 \%)$ of 11 families and lower on the PDI in 9 (82\%) of 11 families. The deficit in PDI appeared more marked than the deficit in MDI. Subjects with CHD had lower mean scores on both the MDI $(85.0 \pm 19.3$ vs $93.9 \pm$ $16.0, P=.037)$ and PDI $(76.6 \pm 16.9$ vs $91.3 \pm 14.9$, $P=.015)$ than their siblings without CHD. On average, subjects with CHD scored 9.7 (95\% confidence interval, 0.7-18.7) points lower on the MDI and 14.7 (95\% con- 
TABLE 2. Additional characteristics of multiple-gestation subjects with CHD

\begin{tabular}{lc}
\hline Parameter & Finding \\
\hline Type of twinning & \\
Fraternal & $8(72.7 \%)$ \\
Unknown & $3(27.3 \%)$ \\
Birth order & \\
One & $6(54.5 \%)$ \\
Two & $5(45.5 \%)$ \\
Median Apgar score at 1 min (range) & $8(4-9)$ \\
Median Apgar score at 5 min (range) & $8(5-9)$ \\
Cardiac diagnosis & \\
TOF & $3(27.3 \%)$ \\
HLHS & $2(18.2 \%)$ \\
D-TGA/IVS & $2(18.2 \%)$ \\
D-TGA/VSD & $1(9.1 \%)$ \\
Critical AS & $1(9.1 \%)$ \\
TAPVR & $1(9.1 \%)$ \\
PA/IVS & $1(9.1 \%)$ \\
Mean CPB duration, min & $72 \pm 45$ \\
DHCA episodes in the first year of life & \\
None & $8(72.7 \%)$ \\
One & $1(9.1 \%)$ \\
Two & $2(18.2 \%)$ \\
Range of DHCA duration, min (single episode) & $15-40$ \\
\hline
\end{tabular}

$C H D$, Congenital heart disease; TOF, tetralogy of Fallot; HLHS, hypoplastic left heart syndrome; $D$-TGA, D-transposition of the great arteries; IVS, intact ventricular septum; VSD, ventricular septal defect; $A S$, aortic stenosis; $T A P V R$, total anomalous pulmonary venous return; $P A$, pulmonary atresia; $C P B$, cardiopulmonary bypass; $D H C A$, deep hypothermic circulatory arrest.

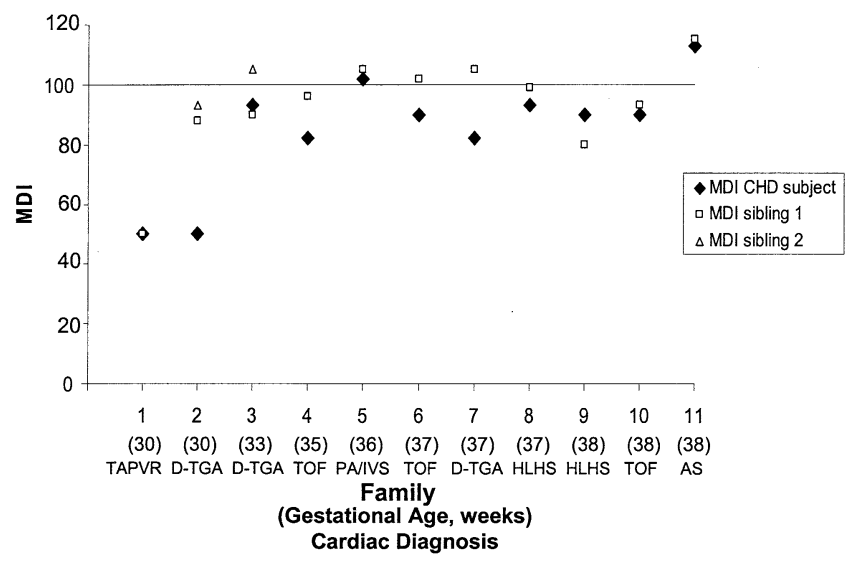

Figure 1. Comparison of Mental Development Index (MDI) scores at 1 year of age within multiple-gestation births. Families are ordered by increasing gestational age at birth. Cardiac diagnosis in the child with congenital heart disease is indicated below gestational age. Families $\mathbf{2}$ and $\mathbf{3}$ are sets of triplets; consequently, results for 2 sibling control subjects are shown. The horizontal line at MDI of 100 represents the population norm. TAPVR, Total anomalous pulmonary venous return; D-TGA, D-transposition of the great arteries; TOF, tetralogy of Fallot; PA, pulmonary atresia; IVS, intact ventricular septum; HLHS, hypoplastic left heart syndrome; $A S$, aortic stenosis.

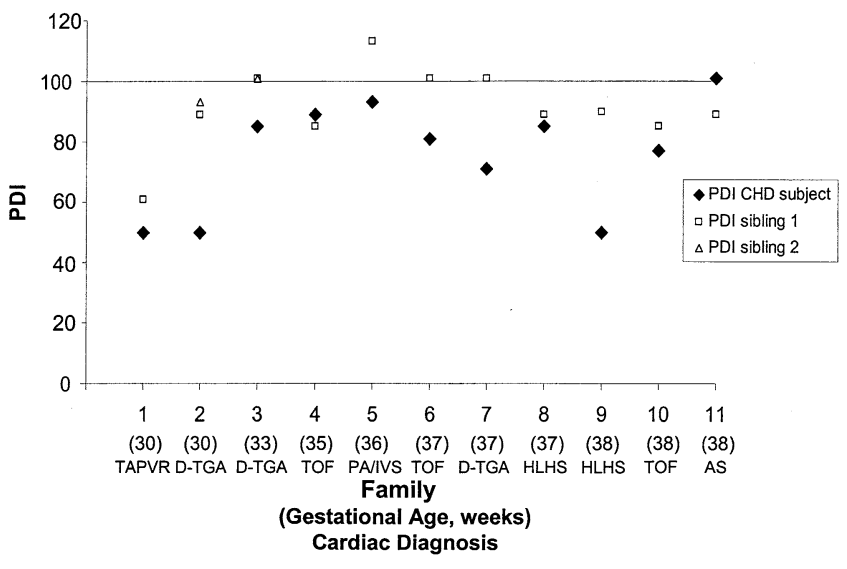

Figure 2. Comparison of Psychomotor Development Index (PDI) scores at 1 year of age within multiple-gestation births. Families are ordered by increasing gestational age at birth. Cardiac diagnosis in the child with congenital heart disease is indicated below gestational age. Families $\mathbf{2}$ and $\mathbf{3}$ are sets of triplets; consequently, results for $\mathbf{2}$ sibling control subjects are shown. The horizontal line at PDI of 100 represents the population norm. TAPVR, Total anomalous pulmonary venous return; D-TGA, D-transposition of the great arteries; TOF, tetralogy of Fallot; $P A$, pulmonary atresia; IVS, intact ventricular septum; HLHS, hypoplastic left heart syndrome; $A S$, aortic stenosis.

fidence interval, 3.4-25.9) points lower on the PDI. No difference in growth parameters or the frequency of an abnormal neurologic examination at 1 year of life was apparent. Mean $z$ scores for the subjects and siblings combined were -0.93 for weight, -0.86 for length, and +0.84 for head circumference.

\section{Discussion}

This study demonstrates that among a cohort of multiplegestation births, the presence of CHD requiring surgical intervention with CPB in the first 6 months of life is associated with lower scores on the Bayley Scales of Infant Development II at 1 year of age. The effect on PDI appears to be more marked than the effect on MDI, which is consistent with prior studies of neurodevelopmental outcomes after infant cardiac surgery. ${ }^{4,9,12,13}$ No difference was seen in somatic growth or the frequency of an abnormal neurologic examination at 1 year of age between children with CHD and their siblings. The pattern of $z$ scores seen for the growth parameters, namely higher mean $z$ scores for head circumference compared with weight or height, is not surprising for a cohort containing a significant fraction of premature infants, in whom head growth catches up earlier than length or weight.

Rather than comparing MDI and PDI scores with population norms, this study used comparisons within multiplegestation births to control optimally for SES, parental intel- 
TABLE 3. Comparison of results of 1-year evaluation

\begin{tabular}{lccc}
\hline Parameter & Subjects with CHD (n= 11) & Siblings without CHD (n = 13) & $P$ value \\
\hline MDI & $85.0 \pm 19.3$ & $93.9 \pm 16.0$ & .037 \\
PDI & $76.6 \pm 16.9$ & $91.3 \pm 14.9$ & .015 \\
Weight (kg) & $8.97 \pm 0.80$ & $9.08 \pm 0.90$ & .71 \\
Length (cm) & $71.9 \pm 2.7$ & $72.7 \pm 2.3$ & .32 \\
Head circumference (cm) & $46.8 \pm 1.4$ & $46.9 \pm 1.4$ & \\
Neurologic examination & $7(63.6 \%)$ & $12(92.3 \%)$ & .85 \\
$\quad$ Normal & $1(9.1 \%)$ & 0 & .18 \\
Suspect & $3(27.3 \%)$ & $1(7.7 \%)$ \\
$\quad$ Abnormal & & \\
\hline
\end{tabular}

$C H D$, Congenital heart disease; $M D I$, Mental Development Index; PDI, Psychomotor Development Index.

ligence, gestational age, and home environment. These potential confounders have been shown to have large effects on cognitive outcomes, effects much greater in magnitude than many interventions studied. For example, social class explained $23.7 \%$ of the variance in full-scale IQ scores at 8 years of age in the Boston Circulatory Arrest Trial, whereas assignment to intraoperative support strategy explained only $0.3 \%$. ${ }^{1}$ A meta-analysis of twin studies has demonstrated that the heritability of IQ is approximately $50 \%$, whereas in utero environment (20\%) and shared postnatal environmental factors (17\%) also contribute importantly to explaining variance in IQ. ${ }^{5,6}$ Mean cognitive scores of children born prematurely decrease in a linear fashion with decreasing birth weight. ${ }^{8}$ Having controlled for these confounders, we observed that children with CHD had deficits in developmental achievement at 1 year of age when compared with their siblings.

The deficits in achievement were observed despite the fact that the majority of the multiple-gestation subjects had CHD that could be corrected to a biventricular circulation in one operation without the use of circulatory arrest. Only 3 children in the study cohort did not have a biventricular repair. The small number of single-ventricle lesions precluded examining the relationship between CHD class and neurodevelopmental outcome. Many of the subjects had the dual vulnerability of both prematurity and CHD. More than half of the subjects required an operation in the neonatal period, which an analysis of the large cohort from which this study population was drawn has suggested might be a period of particular vulnerability to the development of periventricular leukomalacia after CPB. ${ }^{14}$ These observations support the hypothesis that neurodevelopmental impairment after infant cardiac surgery is multifactorial in cause. Research in this field needs to address the complex interaction between multiple factors to optimize patient outcomes.

There are limitations to this study. The mechanisms by which CHD and its management are associated with deficits in developmental outcome at 1 year of age cannot be de- termined by this study. In particular, the effects of CHD, per se, and surgical intervention with CPB cannot be separated from each other. The sample size of the study was small and therefore did not allow precise determination of the magnitude of the difference in MDI and PDI scores.

Correlations between scores on the MDI and PDI at 1 year of age and subsequent achievement are imperfect. In a recent analysis of subjects with D-transposition of the great arteries enrolled in the Boston Circulatory Arrest Trial, correlations between MDI or PDI and achievement or IQ testing at 8 years of age were statistically significant but modest in magnitude (0.16-0.33). ${ }^{15}$ Sensitivity of low MDI or PDI scores for low achievement or IQ scores was poor $(16 \%-31 \%)$, but specificity was good (82\%-93\%). Thus the Bayley Scales of Infant Development provide an early assessment of neurodevelopmental status with some correlation with school age performance, but later follow-up is needed. Follow-up of the multiple-gestation cohort at 4 years of age is currently ongoing.

Given that $4(27 \%)$ of 15 potentially eligible multiplegestation families did not complete follow-up at 1 year, bias could be introduced into the results if those who did return were more or less likely to have neurodevelopmental delay. Although we cannot assess the development of those who did not return, most of the reasons for failure to return seem unlikely to be related to the child's neurodevelopmental status (distance, acute illness, or error on the part of the study staff). The CHD was more severe among these 4 families (3/4 with HLHS or variants) than among those who completed follow-up, making it improbable that inclusion of these families would have reduced the magnitude of the difference observed. Another potential source of bias is that the investigators performing the 1-year evaluation were not blinded to CHD status. This knowledge could have influenced their ratings of subjects' performance on the Bayley Scales and would be predicted to inflate the effect of CHD on neurodevelopmental outcome.

Although the main strength of the study design is the ability to address important confounders, it is possible that 
residual confounding exists. The sibling control subjects were not consistently evaluated by a geneticist. However, underdiagnosis of genetic syndromes in the siblings would tend to bias the results toward the null hypothesis, and the true magnitude of the differences in MDI and PDI would be expected to be larger than reported here. It is possible that genetic syndromes remain undiagnosed among the children with CHD. We have attempted to minimize this possibility by including a formal genetic evaluation at the 1-year visit.

The cohort studied is more premature and of higher SES than a large cohort of children undergoing infant surgical intervention for CHD at the same institution. There might also be independent effects of multiple gestation, particularly among triplets, ${ }^{16}$ on neurodevelopmental outcomes. Thus the ability to generalize these findings to singleton term infants with CHD may be limited. Still, our findings are consistent with patterns observed when cohorts of predominantly singleton term infants are compared with population norms. ${ }^{4,12}$

\section{Summary}

Within multiple-gestation births, children with CHD requiring surgical intervention with CPB in the first 6 months of life have lower scores on the Bayley Scales of Infant Development II at 1 year of age than their siblings without CHD. By using multiple-gestation births, we have optimally controlled for SES, parental intelligence, home environment, and prematurity. Our findings are consistent with those of other studies that have shown developmental deficits among children with CHD compared with general population norms. We observed these deficits despite the fact that the majority of subjects with CHD could be corrected to a biventricular circulation in one operation without the use of DHCA. These results underscore the need for more in-depth understanding of a variety of risk factors for neurodevelopmental impairment to promote optimal outcomes among children with CHD requiring surgical intervention in infancy. Detailed follow-up of this cohort at 4 years of age is currently ongoing.

\section{References}

1. Bellinger DC, Wypij D, du Plessis AJ, Rappaport LA, Jonas RA, Wernovsky G, et al. Neurodevelopmental status at eight years in children with dextro-transposition of the great arteries: the Boston Circulatory Arrest Trial. J Thorac Cardiovasc Surg. 2003;126: 1385-96.

2. Wernovsky G, Stiles KM, Gauvreau K, Gentles TL, duPlessis AJ, Bellinger DC, et al. Cognitive development after the Fontan operation. Circulation. 2000;102:883-9.

3. Mahle WT, Clancy RR, Moss EM, Gerdes M, Jobes DR, Wernovsky G. Neurodevelopmental outcome and lifestyle assessment in schoolaged and adolescent children with hypoplastic left heart syndrome. Pediatrics. 2000;105:1082-9.

4. Gaynor JW, Gerdes M, Zackai EH, Bernbaum J, Wernovsky G, Clancy RR, et al. Apolipoprotein E genotype and neurodevelopmental sequelae of infant cardiac surgery. J Thorac Cardiovasc Surg. 2003; 126:1736-45.

5. Devlin B, Daniels M, Roeder K. The heritability of IQ. Nature. 1997;388:468-71.

6. Bouchard TJ Jr, McGue M. Genetic and environmental influences on human psychological differences. J Neurobiol. 2003;54:4-45.

7. Petrill SA, Deater-Deckard K. Task orientation, parental warmth and SES account for a significant proportion of the shared environmental variance in general cognitive ability in early childhood: evidence from a twin study. Dev Sci. 2004;7:25-32.

8. Bhutta AT, Cleves MA, Casey PH, Cradock MM, Anand KJ. Cognitive and behavioral outcomes of school-aged children who were born preterm: a meta-analysis. JAMA. 2002;288:728-37.

9. Forbess JM, Visconti KJ, Hancock-Friesen C, Howe RC, Bellinger DC, Jonas RA. Neurodevelopmental outcome after congenital heart surgery: results from an institutional registry. Circulation. 2002;106: 95-102.

10. Hollingshead A. Four factor index of social status. New Haven, Conn: Department of Sociology, Yale University; 1975.

11. Clancy RR, McGaurn SA, Wernovsky G, Spray TL, Norwood WI, Jacobs ML, et al. Preoperative risk-of-death prediction model in heart surgery with deep hypothermic circulatory arrest in the neonate. $J$ Thorac Cardiovasc Surg. 2000;119:347-57.

12. Bellinger DC, Jonas RA, Rappaport LA, Wypij D, Wernovsky G, Kuban KC, et al. Developmental and neurologic status of children after heart surgery with hypothermic circulatory arrest or low-flow cardiopulmonary bypass. $N$ Engl J Med. 1995;332:549-55.

13. Jonas RA, Wypij D, Roth SJ, Bellinger DC, Visconti KJ, du Plessis AJ, et al. The influence of hemodilution on outcome after hypothermic cardiopulmonary bypass: results of a randomized trial in infants. J Thorac Cardiovasc Surg. 2003;126:1765-74.

14. Galli KK, Zimmerman RA, Jarvik GP, Wernovsky G, Kuypers MK, Clancy RR, et al. Periventricular leukomalacia is common after neonatal cardiac surgery. J Thorac Cardiovasc Surg. 2004;127: 692-704.

15. McGrath E, Wypij D, Rappaport LA, Newburger JW, Bellinger DC. Prediction of IQ and achievement at age 8 years from neurodevelopmental status at age 1 year in children with D-transposition of the great arteries. Pediatrics. 2004;114:e572-6.

16. Feldman R, Eidelman AI. Does a triplet birth pose a special risk for infant development? Assessing cognitive development in relation to intrauterine growth and mother-infant interaction across the first 2 years. Pediatrics. 2005;115:443-52. 